\title{
On the number of rational squares at fixed distance from a fifth power
}

by

\section{Michael Stoll (Bremen)}

1. Introduction. Let $A \neq 0$ be a rational number. We are interested in the number of rational solutions $(x, y)$ to the equation $y^{2}=x^{5}+A$. In more geometric terms, this amounts to counting the (affine) rational points on the curve $C_{A}$ given by the (affine) equation

$$
C_{A}: y^{2}=x^{5}+A .
$$

In this note, we take up ideas from [St2] and apply them to this family of genus 2 curves. In the following, $C_{A}$ will denote a smooth projective model of the curve in question. With respect to rational points, this means that there is one additional rational point "at infinity", which we will denote $\infty$ in what follows.

Let $J_{A}$ be the Jacobian of $C_{A}$, and denote by $r_{A}$ the Mordell-Weil rank of $J_{A}(\mathbb{Q})$. Since $C_{A}$ and $C_{B}$ are isomorphic when the quotient $A / B$ is a tenth power, we can (and will) assume that $A$ is an integer, not divisible by the tenth power of any prime. Let $n_{A}$ be half the number of "nontrivial" points in $C_{A}(\mathbb{Q})$, i.e., finite points with nonvanishing $x$ and $y$ coordinates. Then $\# C_{A}(\mathbb{Q})=2 n_{A}+d_{A}$, where $d_{A}=1,2,3$, or 4 if $A$ is neither a square nor a fifth power, a fifth power but $A \neq 1$, a square but $A \neq 1$, or $A=1$, respectively. Since $r_{1}=0$, we have $d_{A} \leq 3$ if $r_{A} \geq 1$.

The numbers we are interested in are $n_{A}$ and $\# C_{A}(\mathbb{Q})=d_{A}+2 n_{A}$. The result we will prove is as follows.

THEOREM 1.1. Let $A$ be a tenth power free integer, and assume that $r_{A}=1$. Then $n_{A} \leq 2$ and therefore $\# C_{A}(\mathbb{Q}) \leq 7$. Furthermore, $\# C_{A}(\mathbb{Q})=7$ only when $A=18^{2}$.

2000 Mathematics Subject Classification: Primary 11D41, 11D45, 11G30, 14G05, 14G25; Secondary 11G10, 14H25, 14H40. 
Let $S$ be the set of tenth power free integers. If we define

$$
\begin{aligned}
& N(r)=\max \left\{n_{A}: A \in S, r_{A}=r\right\}, \\
& B(r)=\max \left\{\# C_{A}(\mathbb{Q}): A \in S, r_{A}=r\right\},
\end{aligned}
$$

then the theorem says that $N(1)=2, B(1)=7$.

For $r=0$, we obtain $N(0)=1, B(0)=4$. This is because the torsion points on $C_{A}$ are known to be (see for example [Poo])

$$
\infty, \quad\left(-\zeta^{k} \sqrt[5]{A}, 0\right), \quad(0, \pm \sqrt{A}), \quad\left(\zeta^{k} \sqrt[5]{4 A}, \pm \sqrt{5 A}\right)
$$

where $\zeta$ is a primitive fifth root of unity. The only nontrivial points in this list are of the form given last. But there is only one value of $A$, namely $A=2^{8} \cdot 5^{5}$, such that this leads to a pair of rational points on $C_{A}$. We have $r_{A}=0$ and $n_{A}=1$ in this case (but $d_{A}=1$, so $\# C_{A}(\mathbb{Q})=3$ ). For all other $A$ such that $r_{A}=0$, we must have $n_{A}=0$, hence $\# C_{A}(\mathbb{Q})=d_{A} \leq 4$. The maximum is attained for the unique $A$ with $d_{A}=4$, namely $A=1$.

Since the method of proof can be applied only when $r_{A}<g\left(C_{A}\right)=2$, we cannot obtain exact values for higher ranks. However, we have found the following examples, thus obtaining lower bounds:

\begin{tabular}{|ccccc|}
\hline$r$ & $N(r)$ & $B(r)$ & $A$ with max. N(r) & $A$ with max. $B(r)$ \\
\hline 0 & 1 & 4 & $2^{8} \cdot 5^{5}$ & 1 \\
1 & 2 & 7 & $2^{2} \cdot 3^{4}$ & $2^{2} \cdot 3^{4}$ \\
2 & $\geq 3$ & $\geq 9$ & $2^{2} \cdot 3^{4} \cdot 7^{4}$ & $2^{2} \cdot 3^{4} \cdot 7^{4}$ \\
3 & $\geq 4$ & $\geq 11$ & $2^{2} \cdot 3^{2} \cdot 5^{4} \cdot 7^{4}$ & $2^{2} \cdot 3^{2} \cdot 5^{4} \cdot 7^{4}$ \\
4 & $\geq 6$ & $\geq 15$ & $3^{4} \cdot 7^{4} \cdot 19^{4}$ & $3^{4} \cdot 7^{4} \cdot 19^{4}$ \\
\hline
\end{tabular}

For these examples, the rank $r_{A}$ was determined by first computing an upper bound using 2-descent as described in [St1] and then exhibiting sufficiently many independent points in the Mordell-Weil group (which are here provided by the rational points on $C_{A}$ ).

2. The method. We will apply Chabauty's method with a twist, as explained in [St2]. In that paper, we were only considering sufficiently large primes. In our situation, we obtain the following result in this way. Here and subsequently, $v_{p}$ denotes the normalized $p$-adic valuation.

Proposition 2.1. Suppose that $r_{A}=1$. Then $n_{A} \leq 1$ in the following cases:

(1) $v_{p}(A)=5$ for some prime $p \geq 7$.

(2) $v_{p}(A) \in\{2,4,6,8\}$ for some prime $p \geq 11$.

(3) $v_{p}(A) \in\{1,3,7,9\}$ for some prime $p \geq 17$. 
Proof. We use the main result of [St2], applied to $C_{A}$ with $\Gamma$ taken to be (1) $\mu_{2}$ acting on $y,(2) \mu_{5}$ acting on $x$, and (3) $\mu_{2} \times \mu_{5}$ acting on $(y, x)$, respectively. Note that in each case, $C_{A}$ is a $\Gamma$-twist of $C_{A / p^{v}(A)}$, which has good reduction at $p$.

In principle, this reduces the cases we have to check to a finite number. However, the number of cases is large (a priori, there are $10^{3} \cdot 9 \cdot 5^{2}=225000$ curves; we can expect close to half of them to have $r_{A}=1$ ), and dealing with them one by one would require a very large amount of computation.

We therefore want to use the method at the small primes as well. This will reduce the cases we have to look at to a manageable number.

The basic setup is as follows. If $r_{A}=1$, then there is a differential

$$
\omega=\frac{(\alpha+\beta x) d x}{2 y} \in \Omega\left(C_{A} / \mathbb{Q}_{p}\right)
$$

(with $(\alpha: \beta) \in \mathbb{P}^{1}\left(\mathbb{Q}_{p}\right)$ ) killing the Mordell-Weil group in the sense that

$$
\lambda_{\omega}(P)=\int_{0}^{P} \omega=0 \quad \text { for all } P \in J_{A}(\mathbb{Q}) .
$$

Note that the integral is linear both in $P \in J_{A}\left(\mathbb{Q}_{p}\right)$ and in $\omega$, and vanishes when $P$ is a torsion point in $J_{A}\left(\mathbb{Q}_{p}\right)$. We embed $C_{A}$ into $J_{A}$ using the point at infinity as a base point and from now on consider $C_{A}$ as a subvariety of $J_{A}$. Then $C_{A}(\mathbb{Q})$ is contained in the set of zeros of $\lambda_{\omega}$ on $C_{A}\left(\mathbb{Q}_{p}\right)$. Therefore the number of nontrivial rational points is bounded by the number of nontrivial zeros of $\lambda_{\omega}$ (note that $\lambda_{\omega}$ vanishes at the trivial points, since they are mapped to torsion points of $J_{A}$ ).

Before we use the twisting trick of [St2], let us prove a result that will help reduce the number of cases later, using the standard Chabauty technique.

Lemma 2.2. Assume that $r_{A}=1$. If $A \equiv 1 \bmod 3$, then we have $n_{A} \leq 1$. If $A \equiv-1 \bmod 3$, then we have $n_{A} \leq 2$.

Proof. If $A \equiv 1 \bmod 3$, then $C_{A}\left(\mathbb{F}_{3}\right)=\{\infty,(0,1),(0,-1),(-1,0)\}$. If $A \equiv-1 \bmod 3$, then $C_{A}\left(\mathbb{F}_{3}\right)=\{\infty,(-1,1),(-1,-1),(1,0)\}$.

Let $\bar{\omega}$ be the reduction of (a suitable multiple of) $\omega \bmod 3$. We find that $v(3,0)=0, v(3,1)=1, v(3,2)=0$ in the notation of [St2, $\S 6]$. By Proposition 6.3 in [St2], the number of zeros of $\lambda_{\omega}$ in the residue class of $P \in C_{A}\left(\mathbb{F}_{3}\right)$ is at most $1+n+v(3, n)$, where $n=v_{P}(\bar{\omega})$. This implies that we can only get nontrivial points in residue classes on which $\bar{\omega}$ vanishes, or in nontrivial residue classes. Furthermore, the number of zeros of $\lambda_{\omega}$ in the residue class of $P$ can be at most 3 , since $v_{P}(\bar{\omega}) \leq 2$. The two nontrivial classes $(-1, \pm 1)$ that occur for $A \equiv-1 \bmod 3$ contain the torsion point $(\sqrt[5]{4 A}, \pm \sqrt{5 A}) \in C_{A}\left(\mathbb{Q}_{3}\right)$. Since this point is rational only when $A=2^{8} \cdot 5^{5}$, 
and $r_{A}=0$ in this case, it is still true that we can get nontrivial rational points in these classes only when $\bar{\omega}$ vanishes there.

Let us consider the various classes in turn. If $P=\infty$ or $P=( \pm 1,0)$ and $\bar{\omega}$ vanishes at $P$ (then of second order), there can only be one pair of nontrivial rational points in this residue class, and hence $n_{A} \leq 1$, since no other class contributes to $n_{A}$.

In the other cases, $\bar{\omega}$ can only vanish to first order. One of the a priori up to three zeros on the residue class will be a torsion point, which is trivial or not rational. Therefore there can be at most two pairs of nontrivial rational points, and $n_{A} \leq 2$. It remains to show that in fact, $n_{A} \leq 1$ if $A \equiv 1 \bmod 3$. In this case, we have $P=(0, \pm 1)$ with $v_{P}(\bar{\omega})=1$, so $\bar{\omega}=x d x / 2 y$.

We write $A=a^{2}$ with $a \in \mathbb{Z}_{3}^{\times}$such that $(0, a)$ reduces to the point we are considering. As $t=x$ is a uniformizer, we have, taking $\omega=(3 \alpha+x) d x / 2 y$,

$$
\begin{aligned}
\frac{y}{a} & =1+\frac{t^{5}}{2 A}+O\left(t^{10}\right), & \frac{a d x}{y} & =\left(1-\frac{t^{5}}{2 A}+O\left(t^{10}\right)\right) d t, \\
2 a \omega & =\left(3 \alpha+t-\frac{3 \alpha}{2 A} t^{5}+O\left(t^{7}\right)\right) d t, & 2 a \lambda_{\omega} & =3 \alpha t+\frac{t^{2}}{2}-\frac{\alpha}{4 A} t^{6}+O\left(t^{8}\right) .
\end{aligned}
$$

We see that there is only one nontrivial root in $3 \mathbb{Z}_{3}($ with $t \equiv 3 \alpha \bmod 9)$; therefore $n_{A} \leq 1$.

3. Looking at the small primes. Our first goal is to show that $n_{A} \leq 2$ for all $A$ such that $r_{A}=1$, by a detailed study of the 3 -adic situation. We have covered the case $v_{3}(A)=0$ in Lemma 2.2 already, so here we will consider the cases $1 \leq v_{3}(A) \leq 9$.

We keep the notations introduced above. However, we now suppose that $A$ is divisible by $p$ (we will mostly take $p=3$ below), so that $A=p^{\nu} a$ with some $1 \leq \nu \leq 9$ and $a \in \mathbb{Z}_{p}^{\times}$. Let $\pi=p^{1 / 10}$ and set $x=\pi^{2 \nu} X, y=\pi^{5 \nu} Y$; then over $\mathbb{Q}_{p}(\pi), C_{A}$ is isomorphic to

$$
C_{a}: Y^{2}=X^{5}+a,
$$

and on $C_{a}$,

$$
\omega=\left(\pi^{-\nu} \alpha+\pi^{\nu} \beta X\right) \frac{d X}{2 Y} .
$$

Since $\mathbb{Q}_{p}(\pi) / \mathbb{Q}_{p}$ is totally ramified, the residue class field of $\mathbb{Q}_{p}(\pi)$ is $\mathbb{F}_{p}$. The points in $C_{A}\left(\mathbb{Q}_{p}\right)$ are mapped to one of the following types of points in $C_{a}\left(\mathbb{F}_{p}\right)$ (here $\bar{b} \in \mathbb{F}_{p}$ denotes the image of $b \in \mathbb{Z}_{p}$ ):

1. $\infty$,

2. $(-\bar{b}, 0)$ if $\nu=5$ and $a=b^{5}$ for some $b \in \mathbb{Z}_{p}$,

3. $(0, \bar{b})$ if $\nu$ is even and $a=b^{2}$ for some $b \in \mathbb{Z}_{p}$.

This holds when $p \neq 2,5$. It still holds when $p=2$ or 5 and $p \nmid \nu$. 
We want to bound the number of nontrivial points in $C_{A}(\mathbb{Q})$ mapping to each of these points mod $\pi$. If $p$ is large enough, this bound is given by the order of vanishing of the differential $\bar{\omega}$ at the point in question (assuming $\omega$ is scaled such that it is integral and reduces to something nonzero $\bmod \pi$ ). This is how the results in Proposition 2.1 are obtained. In order to get bounds when $p$ is small, we need to take a closer look at the logarithm

$$
\lambda_{\omega}(T):=\lambda_{\omega}(P(T))=\int_{0}^{P(T)} \omega=\int_{P(0)}^{P(T)} \omega
$$

(recall that the logarithm vanishes on torsion points), where $T$ is a uniformizer at the trivial point $P(0)$ in the residue class under consideration, and $P(T)$ is the point corresponding to the value $T \in \pi \mathcal{O}_{\pi}$ of the uniformizer. This logarithm $\lambda_{\omega}$ can be expanded into a power series in $T$, and the number of its zeros in $\pi \mathcal{O}_{\pi}$ can be bounded above by considering the valuations of the coefficients (and, in some cases, the factoring of polynomials over $\mathbb{F}_{p}$ ). In fact, we are only interested in zeros that arise from points in $C_{A}\left(\mathbb{Q}_{p}\right)$, which restricts the possibilities further.

We discuss the various possible image points in turn.

The residue class of $\infty$. The corresponding points on $C_{A}$ have $t=$ $x^{2} / y \in \mathbb{Z}_{p}$, and therefore have $T=X^{2} / Y=\pi^{\nu} t \in \pi^{\nu} \mathbb{Z}_{p}$. Note that $T$ is a uniformizer at $\infty$ on $C_{a}$. We have the equations

$$
X^{-1}=T^{2}\left(1+a X^{-5}\right), \quad Y^{-1}=T X^{-2},
$$

so

$$
\begin{aligned}
X & =T^{-2}\left(1-a T^{10}+O\left(T^{20}\right)\right), \\
-\frac{d X}{2 Y} & =T^{2}\left(1+6 a T^{10}+O\left(T^{20}\right)\right) d T, \\
-\omega & =\left(\beta \pi^{\nu}+\alpha \pi^{-\nu} T^{2}+5 a \beta \pi^{\nu} T^{10}+6 a \alpha \pi^{-\nu} T^{12}+O\left(T^{20}\right)\right) d T, \\
-\lambda_{\omega} & =\beta \pi^{\nu} T+\frac{\alpha}{3} \pi^{-\nu} T^{3}+\frac{5 a \beta}{11} \pi^{\nu} T^{11}+\frac{6 a \alpha}{13} \pi^{-\nu} T^{13}+O\left(T^{21}\right) \\
& =\pi^{2 \nu}\left(\beta t+\frac{\alpha}{3} t^{3}+\frac{5 a \beta}{11} p^{\nu} t^{11}+\frac{6 a \alpha}{13} p^{\nu} t^{13}+\cdots\right) .
\end{aligned}
$$

Since 3, 11 and 13 are the only primes occurring in the denominators of relevant coefficients (the later terms do not matter, as is easily seen), we see that for all other primes $p$, the following holds.

If $\overline{(\alpha: \beta)}=(0: 1) \in \mathbb{P}^{1}\left(\mathbb{F}_{p}\right)$, then there is only one rational point $($ namely $\infty)$ in this residue class. Otherwise, if $\overline{(\alpha: \beta)}=(1: 0)$, there may be three, and if $\overline{(\alpha: \beta)}=(1: \xi)$ with $\xi \neq 0$, there is one point if $-3 \alpha \beta$ is a nonsquare $\bmod p$, and at most three points if $-3 \alpha \beta$ is a square $\bmod p$. 
We will not discuss $p=11$ and $p=13$ here. If $p=3$, there always are at most three points, but the condition is shifted. We write

$$
\omega=\left(3 \alpha^{\prime}+\beta x\right) d x / 2 y \quad \text { with } \alpha^{\prime}, \beta \in \mathbb{Z}_{3} ;
$$

then there can be three points if $\bar{\alpha}^{\prime} \neq 0$ and $-\bar{\alpha}^{\prime} \bar{\beta}$ is a square. We obtain the following result, strengthening Proposition 2.1(3).

Lemma 3.1. Suppose $r_{A}=1$. If $v_{p}(A) \in\{1,3,7,9\}$ for some $p \neq 11,13$, then $n_{A} \leq 1$.

Proof. Note that if $v_{p}(A) \in\{1,3,7,9\}$, then $\infty$ is the only point in $C_{a}\left(\mathbb{F}_{p}\right)$ that is hit by $C_{A}(\mathbb{Q})$. By the preceding discussion, there is at most one pair of nontrivial rational points in this residue class.

The residue class of $(-b, 0)$. We now assume $\nu=5$ (and $p \neq 5$ ) and $a=b^{5}$, so we have $y^{2}=x^{5}+p^{5} b^{5}$. The points in the residue class we are considering have $x \equiv-b p \bmod p^{2}$ and $y \equiv 0 \bmod p^{3}$. We choose $T=Y$ as the uniformizer on $C_{a}$; then $T=\sqrt{p} t$ with $t \in \mathbb{Z}_{p}$. Expanding everything in terms of $T$, we get

$$
\begin{aligned}
X & =-b\left(1-a^{-1} T^{2}\right)^{1 / 5} \\
& =-b\left(1-\frac{1}{5 a} T^{2}-\frac{2}{(5 a)^{2}} T^{4}-\frac{6}{(5 a)^{3}} T^{6}+O\left(T^{8}\right)\right), \\
\frac{5 a}{b} \frac{d X}{2 Y} & =\left(1+\frac{4}{5 a} T^{2}+\frac{18}{(5 a)^{2}} T^{4}+O\left(T^{8}\right)\right) d T \\
\frac{5 a}{b} \frac{X d X}{2 Y} & =-b\left(1+\frac{3}{5 a} T^{2}+\frac{12}{(5 a)^{2}} T^{4}+O\left(T^{8}\right)\right) d T \\
\frac{5 a}{b} \omega & =\frac{1}{\sqrt{p}}\left((\alpha-b \beta p)+\frac{4 \alpha-3 b \beta p}{5 a} T^{2}+\cdots\right) d T \\
\frac{5 a}{b} \lambda_{\omega} & =\frac{1}{\sqrt{p}}(\alpha-b \beta p) T+\frac{4 \alpha-3 b \beta p}{3 \cdot 5 a} T^{3}+\cdots \\
& =(\alpha-b \beta p) t+\frac{(4 \alpha-3 b \beta p) p}{3 \cdot 5 a} t^{3}+\cdots .
\end{aligned}
$$

The interesting case for us here is $p=3$. We again write $\alpha=3 \alpha^{\prime}$ and assume that $\alpha^{\prime}, \beta \in \mathbb{Z}_{3}$, not both in $3 \mathbb{Z}_{3}$; then up to scaling, $\bmod 3$ we have

$$
\lambda_{\omega} \sim t\left(\left(\bar{\alpha}^{\prime}-\bar{b} \bar{\beta}\right)-\bar{a}^{-1} \bar{\alpha}^{\prime} t^{2}\right) .
$$

If $\bar{\alpha}^{\prime}=0$, there will be only one solution. If $\alpha^{\prime}=1$, we will have three solutions if $a(1-b \beta)$ is a square, and one solution if it is a nonsquare. In any case, there is at most one pair of nontrivial rational points in this residue class. For all other primes $p \neq 5$, we get at most one pair of nontrivial rational points in this class as well, but only if $v_{p}(\alpha)>v_{p}(\beta)$. We therefore obtain, taking into account the discussion of $\infty$ above, the following strengthening of Proposition 2.1(1). 
Lemma 3.2. Suppose $r_{A}=1$. If $v_{3}(A)=5$, then $n_{A} \leq 2$. If $v_{p}(A)=5$ for a prime $p \notin\{3,5\}$, then $n_{A} \leq 1$.

The residue class of $(0, b)$. Let $\nu=2 n$ and set $\mu=\min \{m \in \mathbb{Z}: 5 m>n\}$. We must exclude $p=2$ here. We have $\varrho=5 \mu-\nu=3,1,4,2$ for $n=1,2,3,4$, respectively. The points in the residue class have $x=p^{\mu} t$ with $t \in \mathbb{Z}_{p}$ and $y \equiv b p^{n} \bmod p^{n+1}$. We choose $T=X=\pi^{2 \varrho} t$ as a uniformizer on $C_{a}$. This gives

$$
\begin{aligned}
Y^{-1} & =b^{-1}\left(1+a^{-1} T^{5}\right)^{-1 / 2}=b^{-1}\left(1-\frac{1}{2 a} T^{5}+\frac{3}{8 a^{2}} T^{10}+O\left(T^{15}\right)\right), \\
b \frac{d X}{Y} & =\left(1-\frac{1}{2 a} T^{5}+\frac{3}{8 a^{2}} T^{10}+O\left(T^{15}\right)\right) d T \\
2 b \omega & =\left(\alpha \pi^{-2 n}+\beta \pi^{2 n} T-\frac{\alpha}{2 a} \pi^{-2 n} T^{5}-\frac{\beta}{2 a} \pi^{2 n} T^{6}+O\left(T^{10}\right)\right) d T, \\
2 b \lambda_{\omega} & =\alpha \pi^{-2 n} T+\frac{\beta}{2} \pi^{2 n} T^{2}-\frac{\alpha}{3 \cdot 4 a} \pi^{-2 n} T^{6}-\frac{\beta}{7 \cdot 2 a} \pi^{2 n} T^{7}+\cdots \\
& =\pi^{2(\varrho-n)}\left(\alpha t+\frac{\beta}{2} p^{\mu} t^{2}-\frac{\alpha}{3 \cdot 4 a} p^{\varrho} t^{6}-\frac{\beta}{7 \cdot 2 a} p^{\varrho+\mu} t^{7}+\cdots\right) .
\end{aligned}
$$

If $p \notin\{2,3,7\}$, then there is at most one nontrivial solution, and we obtain at most one pair of nontrivial rational points mapping to $(0, \pm \bar{b})$. Together with the discussion of $\infty$, this proves the following. The only new case is $p=5$, since $p \geq 11$ is already taken care of by Proposition 2.1(2).

Lemma 3.3. Assume $r_{A}=1$ and $v_{p}(A) \in\{2,4,6,8\}$ for some $p \notin$ $\{2,3,7\}$. Then $n_{A} \leq 1$.

Proof. We have a contribution of at most 1 to $n_{A}$ from $\infty$ and a contribution of at most 1 from $(0, \pm \bar{b})$. However, we get a contribution from $\infty$ only when $v_{p}(\beta) \geq v_{p}(\alpha)$, but then there is no contribution from $(0, \pm \bar{b})$, as can be seen from the expansion of $\lambda_{\omega}$ above.

We have to consider the cases $p=3$ and $p=7$ separately. When $p=3$, we have, with $\omega=\left(3 \alpha^{\prime}+\beta x\right) d x / 2 y$ as before,

$$
\lambda_{\omega} \sim \alpha^{\prime} t+\frac{\beta}{2} 3^{\mu-1} t^{2}-\frac{\alpha^{\prime}}{4 a} 3^{\varrho-1} t^{6}+\cdots .
$$

If $\varrho>1$, only the first two terms matter. We get extra solutions only if $v_{3}\left(\alpha^{\prime}\right) \geq v_{3}(\beta)+\mu-1$. If $\varrho=1$ (and therefore $\mu=1, \nu=4$ ), we have to look at solutions in $\mathbb{F}_{3}$ of

$$
t\left(\bar{\alpha}^{\prime}-\bar{\beta} t-\bar{\alpha}^{\prime} t^{5}\right)
$$

(Recall that $a$ is a square, so $\bar{a}=1$.) If $\bar{\alpha}^{\prime}=0$, there is one extra solution. Otherwise, we can take $\alpha^{\prime}=1$, and then we have no extra solutions if $\bar{\beta}=-1$, we have one extra solution if $\bar{\beta}=0$, and we have potentially two 
extra solutions if $\bar{\beta}=1$. Considering this together with the result at infinity, we get the following.

Lemma 3.4. Suppose $r_{A}=1$. If $v_{3}(A) \in\{6,8\}$, then $n_{A} \leq 1$. If $v_{3}(A) \in$ $\{2,4\}$, then $n_{A} \leq 2$.

Proof. The following table summarizes the possible contributions to $n_{A}$ from the residue classes of $\infty$ and of $(0, \pm b)$, depending on the reduction $\bmod 3$ of $\left(\alpha^{\prime}: \beta\right) \in \mathbb{P}^{1}\left(\mathbb{Q}_{3}\right)$ :

\begin{tabular}{|c||c|ccc|}
\hline \multicolumn{1}{c||}{} & \multirow{3}{|c|}{$(0, \pm b)$} \\
& $\infty$ & $\nu=2$ & $\nu=4$ & $\nu=6,8$ \\
\hline$(0: 1)$ & 0 & $\leq 1$ & $\leq 1$ & $\leq 1$ \\
$(1: 0)$ & $\leq 1$ & 0 & $\leq 1$ & 0 \\
$(1: 1)$ & 0 & $\leq 1$ & $\leq 2$ & 0 \\
$(1:-1)$ & $\leq 1$ & $\leq 1$ & 0 & 0 \\
\hline
\end{tabular}

We see that $n_{A} \leq 1$ for $\nu=6$ or 8 , whereas $n_{A} \leq 2$ for $\nu=2$ or 4 .

Now we consider $p=7$. This leads to

$$
\lambda_{\omega} \sim t\left(\alpha+\frac{\beta}{2} 7^{\mu} t-\frac{\alpha}{12 a} 7^{\varrho} t^{5}-\frac{\beta}{2 a} 7^{\varrho+\mu-1} t^{6}+\cdots\right) .
$$

The $t^{5}$ term is irrelevant. If $\bar{\alpha} \neq 0$, then there are no extra solutions. In general, we need $v_{7}(\alpha) \geq v_{7}(\beta)+\mu$ for there to be extra solutions. In this case, if $\varrho>1$, then there is just one extra solution. If $\varrho=1$, i.e., $\nu=4$ and $\alpha=7 \alpha^{\prime}$, then (taking $\beta=1$ without loss of generality), we must consider the roots in $\mathbb{F}_{7}$ of

$$
\bar{\alpha}^{\prime} t-3 t^{2}+3 \bar{a}^{-1} t^{7}
$$

There can be more than one extra solution; in this case there are up to four extra solutions. In any case, together with the result at infinity, we get the following.

Lemma 3.5. Suppose $r_{A}=1$. If $v_{7}(A) \in\{2,6,8\}$, then $n_{A} \leq 1$.

Putting it together. Collecting the information obtained so far, we see that Lemmas 2.2, 3.1, 3.2 and 3.4 cover all cases. This proves the first part of Theorem 1.1.

Now, if $A$ is such that $r_{A}=1$ and $\# C_{A}(\mathbb{Q})=7$, we need to have $d_{A}=3$ and $n_{A}=2$. So $A$ has to be a square. Furthermore, by Lemma 3.3, the prime factors of $A$ are contained in $\{2,3,7\}$, by Lemma $3.5, v_{7}(A) \in\{0,4\}$, and by Lemmas 2.2 and $3.4, v_{3}(A) \in\{2,4\}$. This leaves $5 \cdot 2 \cdot 2=20$ values of $A$ to check. We can reduce the number of cases further by noting that if $A \equiv 1,3,9 \bmod 11$, all points in $C_{A}\left(\mathbb{F}_{11}\right)$ lift to torsion points in $C_{A}\left(\mathbb{Q}_{11}\right)$, and therefore by standard Chabauty, $n_{A} \leq 1$. This reduces the list to the 
following eight values:

$$
A \in\left\{3^{2} \cdot 7^{4}, 3^{4}, 2^{2} \cdot 3^{4}, 2^{2} \cdot 3^{4} \cdot 7^{4}, 2^{4} \cdot 3^{4} \cdot 7^{4}, 2^{6} \cdot 3^{2}, 2^{8} \cdot 3^{2}, 2^{8} \cdot 3^{2} \cdot 7^{4}\right\} .
$$

The table below summarizes the data for these curves:

\begin{tabular}{|lcc|lcc|}
\hline$A$ & $r_{A}$ & $n_{A}$ & $A$ & $r_{A}$ & $n_{A}$ \\
\hline $3^{2} \cdot 7^{4}$ & 2 & $\geq 1$ & $3^{4}$ & 2 & $\geq 2$ \\
$2^{2} \cdot 3^{4}$ & 1 & 2 & $2^{2} \cdot 3^{4} \cdot 7^{4}$ & 2 & $\geq 3$ \\
$2^{4} \cdot 3^{4} \cdot 7^{4}$ & 3 & $\geq 2$ & $2^{6} \cdot 3^{2}$ & 1 & 1 \\
$2^{8} \cdot 3^{2}$ & 1 & 0 & $2^{8} \cdot 3^{2} \cdot 7^{4}$ & 0 & 0 \\
\hline
\end{tabular}

The ranks $r_{A}$ have been found by computing an upper bound as described in [St1] and exhibiting sufficiently many independent points in $J_{A}(\mathbb{Q})$ (which are mostly provided by points in $\left.C_{A}(\mathbb{Q})\right)$. The values for $n_{A}$ in the cases when $r_{A}=1$ have been verified by a standard Chabauty computation (at $p=29$ for $A=2^{8} \cdot 3^{2}$, at $p=29$ and $p=59$ for $A=2^{6} \cdot 3^{2}$ : there are four extra residue classes left after the computation with $p=29$, which can then be excluded by looking mod 59; see [PSS, §12] for an explanation of the method).

This shows that $A=18^{2}=2^{2} \cdot 3^{4}$ is the only value such that $r_{A}=1$ and $C_{A}$ has 7 rational points. The last statement of Theorem 1.1 is therefore also verified.

It would be interesting to find out if there are more values of $A$ such that $r_{A}=1$ and $n_{A}=2$ (which then will be nonsquares). It is clear by the discussion in this paper that there can only be finitely many. The problem one encounters in practice is that in many cases, generators of the Mordell-Weil group appear to be too large to be found, and hence the Chabauty computation cannot be carried out. On the other hand, it is very unlikely that there are nontrivial points on $C_{A}$ when $J_{A}(\mathbb{Q})$ has a very large generator. Since none of the curves that are amenable to computation has $n_{A}=2, A=18^{2}$ is likely to be the only value of $A$ that has $r_{A}=1$ and $n_{A}=2$.

In any case, it is easy to see that there is no $A$ such that $r_{A}=1$ and $\# C_{A}(\mathbb{Q})=6$. This would imply that $n_{A}=2$ and $A$ is a fifth power, so by Lemma $3.2, A$ is one of $1,3^{5}, 5^{5}, 3^{5} \cdot 5^{5}$. But all these values satisfy $A \equiv 1 \bmod 11$, so $n_{A} \leq 1$ if $r_{A}=1$ (which is the case for $A=3^{5}$ and $3^{5} \cdot 5^{5}$ ).

On the other hand, there are likely to be infinitely many $A$ such that $r_{A}$ $=1$ and $\# C_{A}(\mathbb{Q})=5$ : every $A=a^{2}$ such that $r_{A}=1$ and such that $C_{A}$ has a nontrivial rational point will provide an example (unless $a=18$, of course). Values of $a$ satisfying the latter condition abound (writing $(y+a)(y-a)=x^{5}$, they are easily parametrized), and we can expect infinitely many of them to satisfy $r_{a^{2}}=1$. However, it appears to be very hard to prove that, or even give a reasonable characterization of these values. 


\section{References}

[Poo] B. Poonen, Computing torsion points on curves, Experiment. Math. 10 (2001), 449-465.

[PSS] B. Poonen, E. F. Schaefer and M. Stoll, Twists of X(7) and primitive solutions to $x^{2}+y^{3}=z^{7}$, Duke Math. J., to appear; arXiv:math.NT/0508174.

[St1] M. Stoll, Implementing 2-descent for Jacobians of hyperelliptic curves, Acta Arith. 98 (2001), 245-277.

[St2] -, Independence of rational points on twists of a given curve, Compos. Math. 142 (2006), 1201-1214.

School of Engineering and Science

International University Bremen

P.O. Box 750561

28725 Bremen, Germany

E-mail: m.stoll@iu-bremen.de

Received on 13.3.2006

and in revised form on 10.8.2006 